\title{
Review: evidence is sparse and inconclusive for treating and monitoring chronic mild to moderate hypertension in pregnancy
}

\author{
Mulrow CD, Chiquette E, Ferrer RL, et al. Management of chronic hypertension during pregnancy. Evidence \\ Report/Technology Assessment No. 14. AHRQ Publication No. O0-E011. Rockville, MD:Agency for Healthcare Research and \\ Quality; August 2000.

\section{QUESTIONS: In pregnant women with mild to moderate chronic hypertension, what is the magnitude of maternal and fetal risks, how effective and safe are treatments before and during pregnancy, and what monitoring strategies are effective for detecting fetal complications associated with the hypertension?}

Source of funding: US Agency for Healthcare and Research Quality.

For correspondence: Dr C D Mulrow, Audie L Murphy Veterans Hospital, Quality of Life Projects, 7400 Merton Minter Boulevard, (11C-6), San Antomio, TX 78284, USA.Fax +1 2105674423.

\section{COMMENTARY}

Mulrow et al have provided an excellent 208 page review on the management of chronic hypertension in pregnancy, an important condition that has been neglected for the past half century. The report is more revealing as a model of how any medical subject should be reviewed than it is for its own findings. Most current published evidence presents serious methodological problems, particularly in the study design, lack of statistical power, and scant sample size. 16 electronic sources were searched, and 6228 records were retrieved; only 215 studies met the inclusion criteria.

Some relevant aspects are not included in the review, such as risk factors for mother and fetus within the studied groups, when delivery should occur, effectiveness of treatment of hypertension to prevent severe hypertension, and non-pharmacological means to prevent high blood pressure (BP) and pre-eclampsia. For example, the use of calcium, 1-2 g/day, may prevent pre-eclampsia and increased BP in high risk pregnant women. ${ }^{1}$

The review provides important information on the adverse effects of antihypertensive agents in pregnancy; ACE inhibitors must not be used, although this decision is generally based on poor quality data. Studies on 2 other commonly used drug groups, $B$ blockers and calcium channel blockers, showed conflicting evidence. Methyldopa, the most frequently prescribed drug for hypertension, has been used in just over 500 women in clinical trials. It is sad to observe that such a small amount of evidence has been the basis for treating millions of pregnant women worldwide. No evidence exists to answer the question of when treatment of hypertension should be initiated and at what $\mathrm{BP}$. The authors recognise the association between higher $\mathrm{BP}$ and maternal and fetal risks. Evidence is more conclusive about treatment of patients with severe hypertension ( $\geqslant 170 \mathrm{~mm} \mathrm{Hg}$ systolic or $\geqslant 110 \mathrm{~mm} \mathrm{Hg}$ diastolic BP).

Large collaborative RCTs and prospective cohort studies on chronic hypertension in pregnancy are badly needed. Chronic hypertension increases maternal mortality from 11 to 230/100 000 in the US. ${ }^{2}$ The even higher prevalence of adverse outcomes in developing countries makes these regions suitable for carrying out large, sensitive collaborative RCTs to obtain evidence on how to reduce the burden of the disease. lvaro N Atallah, MD Universidade Federal de São Paulo São Paulo SP Brasil

1 Atallah NA, Hofmeyr GJ, Dulcy L. Calcium supplementation during pregnancy for preventing hypertensive disorders and related problems. Cochrane Database Syst Rev 2001;(1):CD001059.

2 Jain L. Effect of pregnancy-induced and chronic hypertension on pregnancy outcome.J Perinatol 1997;17:425-7. studies for causation and monitoring questions). All included studies evaluated pregnant women with chronic hypertension.

\section{Data extraction}

Data were extracted on study design and quality, patient populations, selection and outcome criteria, interventions and comparisons, and outcomes. Analysis used random effects models.

\section{Main results}

Few clinical data are available to show the benefits of treating chronic hypertension in non-pregnant young women, although a meta-analysis of 3 RCTs of women aged 30-54 years showed that approximately 259 women (95\% CI 158 to 1606) would need to be treated for 5 years to prevent 1 additional cardiovascular event. Data are insufficient to prove or disprove the benefits of treating chronic hypertension in pregnant women. Although data are sparse, several antihypertensive agents have been associated with adverse events in pregnant women. Angiotensin converting enzyme (ACE) inhibitors, if used in the second or third trimester, have been shown to be associated with renal dysfunction in the fetus. Conflicting trial evidence exists on the connection between atenolol and fetal growth retardation. Some anecodotal evidence on nifedipine supports the finding that it causes neuromuscular blockage if it is used in conjunction with magnesium. Diuretics, methyldopa, and hydralazine are safe for mothers and infants. No RCTs have been done on non-pharmacological interventions for mild to moderate hypertension. Although chronic hypertension is associated with a 3 -fold increased risk for perinatal mortality and an increased risk for abruption, pre-eclampsia, and smaller babies, optimal target levels for starting treatment have not been ascertained. Monitoring techniques (biophysical profiles, Doppler velocimetry, non-stress tests, contraction stress tests, fundal measurements, amniotic fluid index, ultrasonographic fetal biometry, or fetal movement counting) have not been shown to identify fetal complications.

\section{Conclusion}

Evidence is sparse and inconclusive on the effects of treating mild to moderate chronic hypertension before or during pregnancy, the adverse effects of antihypertensive agents, and the usefulness of monitoring techniques to identify fetal complications. 